proteins were purified with protein A-Sepharose beads. Proteins were quantitated by dot immunoblot analysis by using conjugated antibody to human IgG-horseradish peroxidase (Jackson ImmunoResearch Lab). Protein purity was analyzed by SDS-polyacrylamide gel electrophoresis (PAGE) and silver staining. Ligand binding specificity of the proteins was assessed with NIH 3T3 cells. Briefly, serum-starved cells were stimulated with FGFs in the presence of either FR1-IgG or FR4-IgG for 5 hours. Total cell lysates were prepared and the changes in the protein phosphorylation profile were determined by immunoblot analysis with an antibody to phosphotyrosine (Santa Cruz).

30. D. M. Ornitz et al., J. Biol. Chem. 271, 15292 (1996); C. A. Dionne et al., EMBO J. 9, 2685 (1990).

31. Data not shown for FGF2.

32. S. Sell, M. A. Longley, J. Boulter, Dev. Br. Res. 22, 49 (1985); A. Poliard, G. Feldman, D. Bernau, Differenti- ation 39, 59 (1988); J. Nahon et al., J. Biol. Chem. 263, 11436 (1988).

33. Supported by National Institutes of Health grant GM36477 to K.S.Z. We thank G. Martin for the FGF CDNAs; K. Matsumoto for FGF2 monoclonal antibody; and J. Coleman, P. Bossard, and J. Lora for comments on the manuscript.

22 February 1999; accepted 13 May 1999

\section{Identification of Both Shared and Distinct Proteins in the Major and Minor Spliceosomes}

\author{
Cindy L. Will, ${ }^{1}$ Claudia Schneider, ${ }^{1}$ Robin Reed, ${ }^{2}$ \\ Reinhard Lührmann ${ }^{1,3 *}$
}

\begin{abstract}
In metazoans, two distinct spliceosomes catalyzing pre-messenger RNA splicing have been identified. Here, the human U11/U12 small nuclear ribonucleoprotein (snRNP), a subunit of the minor (U12-dependent) spliceosome, was isolated. Twenty U11/U12 proteins were identified, including subsets unique to the minor spliceosome or common to both spliceosomes. Common proteins include four U2 snRNP polypeptides that constitute the essential splicing factor SF3b. A 35-kilodalton U11-associated protein homologous to the U1 snRNP 70K protein was also identified. These data provide fundamental information about proteins of the minor spliceosome and shed light on its evolutionary relationship to the major spliceosome.
\end{abstract}

The minor (U12-dependent) spliceosome is required for splicing a rare class of nuclear pre-mRNA introns (1). These so-called U12type introns contain highly conserved sequence elements at the $5^{\prime}$ splice site and branch site that are distinct from the weakly conserved sequence elements found in the major class of pre-mRNA introns $(1,2)$. During assembly of the minor spliceosome, the U11 snRNP forms base pairs with the $5^{\prime}$ splice site, and the U12 snRNP forms base pairs with the branch site, analogous to the $\mathrm{U} 1$ and $\mathrm{U} 2$ snRNPs in the major spliceosome (3). Subsequently, mature spliceosomes are formed by the association of the U5 and U4atac/U6atac snRNPs (4). In the major spliceosome, 5' splice site and branch site recognition are mediated by multiple interactions involving both RNA and protein (5). In metazoans, formation of the U1 snRNA/ $5^{\prime}$ splice site duplex is facilitated by several polypeptides, including the U1 snRNP $70 \mathrm{~K}$ and $\mathrm{C}$ proteins, as well as members of the SR (serine- and arginine-rich) protein family (6). Similarly, base pairing between U2 snRNA and the branch site requires numerous $\mathrm{U} 2$ snRNP proteins, in particular the subunits of

${ }^{1}$ Institut für Molekularbiologie und Tumorforschung, Philipps-Universität, 35037 Marburg, Germany. ${ }^{2}$ Department of Cell Biology, Harvard Medical School, Boston, MA 02115, USA. ${ }^{3}$ Department of Cellular Biochemistry, Max Planck Institute of Biophysical Chemistry, 37070 Göttingen, Germany.

*To whom correspondence should be addressed. Email: luehrmann@imt.uni-marburg.de the heteromeric splicing factors SF3a and SF3b (5-7).

In contrast to their counterparts in the major spliceosome, the U11 and U12 snRNPs are present in nuclear extract not only as individual monoparticles, but also as a highly stable $18 S$ U11/U12 complex (8). Recent in vitro binding studies suggest that U11 and U12 interact with the pre-mRNA as a preformed complex (9). This observation, coupled with the fact that U12-type introns lack the essential pyrimidine tract found at the $3^{\prime}$ splice site of the major class of introns, suggests that differences may exist in the mechanism of initial splice site recognition and pairing in the two types of spliceosomes. To identify proteins involved in this process in the minor spliceosome, we have characterized polypeptides associated with the human $18 S$ U11/U12 snRNP complex.

Spliceosomal snRNPs were immunoaffinity-purified from $\mathrm{HeLa}$ nuclear extract and fractionated by glycerol gradient sedimentation. From the $18 S$ region of the gradient, U11/U12 snRNP complexes were then affinity-selected using biotinylated 2'-O-methyl oligonucleotides and streptavidin agarose (10). snRNPs containing predominantly $\mathrm{U} 11$ and $\mathrm{U} 12$ snRNA were selected by oligonucleotides complementary to nucleotides 2 to 18 of the U11 snRNA (Fig. 1A, lane 2) or nucleotides 11 to 28 of the U12 snRNA (Fig. 1A, lane 3). The coselection of U12 with an oligonucleotide directed against U11, and vice versa, indicated that mainly $18 S$ U11/U12 snRNP complexes (as opposed to U11 or U12 monoparticles) had been selected. Consistent with this conclusion, the U11/U12 snRNPs selected by either oligonucleotide exhibited identical protein patterns (Fig. 1B, lanes 2 and 3). Twenty distinct proteins were detected in the U11/U12 complex. Eight of these comigrated with the snRNP Sm proteins B', B, D3, D2, D1, E, F, and G, which are present in the major spliceosomal snRNPs (Fig. 1B, lanes 1 to 3 ; see also Fig. 2) (11). Antibodies reacting specifically with $\mathrm{B}^{\prime} / \mathrm{B}, \mathrm{D} 3$, $\mathrm{D} 2, \mathrm{~F}$, or $\mathrm{G}$ also recognized proteins of identical molecular mass on immunoblots of the U11/ U12 complex (12). These data indicate that U11/U12 contains the same eight Sm proteins

Table 1. U2 snRNP proteins are also present in U11/U12 snRNPs. $X$ indicates an unidentified amino acid (28). Mismatches (indicated by boldface letters) were observed between the U11/U12-160kD and U2-160kD proteins at position 149 ( $V$ versus $A$ ) and between the U11/U12-130kD and U2-120kD proteins at positions 1209, 1211, and 1212 (D versus $N, R$ versus $A$, and T versus $Q$, respectively).

\begin{tabular}{llccc}
\hline $\begin{array}{c}\text { U11/U12 } \\
\text { protein }\end{array}$ & \multicolumn{1}{c}{ Peptides } & Identity & Reference & Amino acids \\
\hline $160 \mathrm{kD}$ & KMNARTYMDVMREQHLTK & U2-160kD & $(16)$ & $146-162$ \\
& KLTATPTPLGGMTGF & & & $428-442$ \\
& KAIVNVIGMH & & & $997-1007$ \\
$150 \mathrm{kD}$ & KRIFEAFK & U2-150kD & $(17)$ & $364-371$ \\
& KLRRMNRFTVAE & & & $430-441$ \\
& KRTGIQEMREALQEK & & & $506-520$ \\
& KLTIHGDLYYG & & & $560-571$ \\
$130 \mathrm{kD}$ & KLGAVFNQVAFPLQYT & U2-120kD & $(29)$ & $768-783$ \\
& KLLRVYDLGK & & & $964-973$ \\
& KNVSEELDRTPPEVSK & & & $1190-1205$ \\
& KLENIAQRYAF & & $1206-1217$ \\
$49 \mathrm{kD}$ & KVSEPLLXELFLQ & U2-53kD & $(18)$ & $23-35$ \\
& KDRVTGQHQGYGFVFLSEE & & & $46-65$ \\
\hline
\end{tabular}




\section{R E P O R T S}
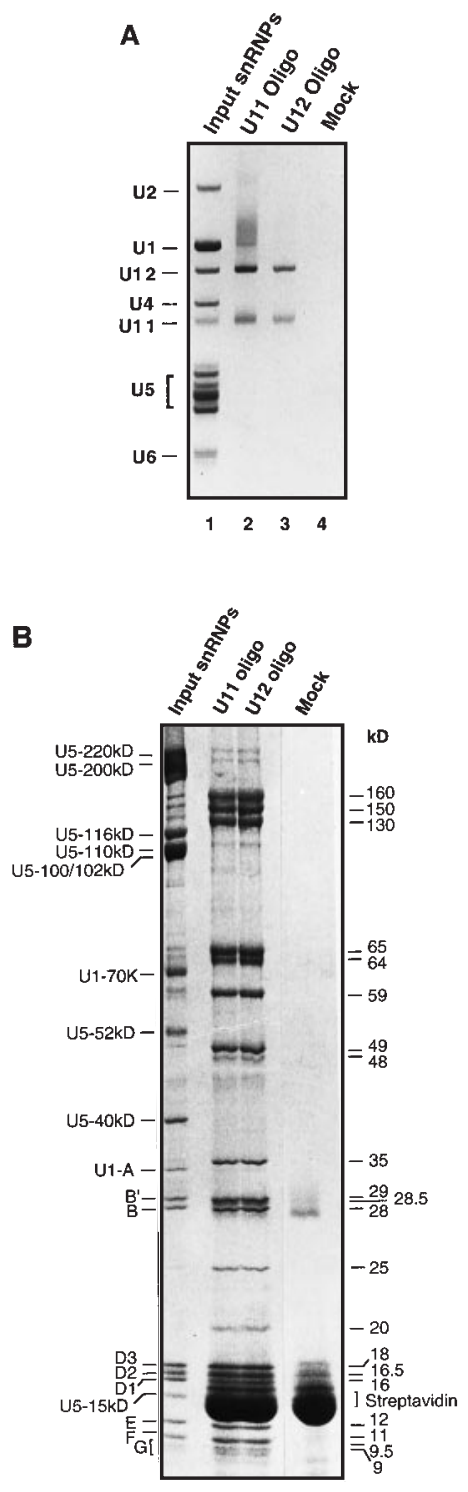

Fig. 1. Proteins associated with $18 S$ U11/U12 snRNP complexes. (A) snRNA composition of purified snRNPs. snRNAs were isolated from snRNPs precipitated in the absence (Mock) or presence of the indicated oligonucleotide. (B) Protein composition of oligonucleotide-selected U11/U12 snRNPs. Molecular masses of the U11/U12 proteins are shown on the right, and the identity of the major proteins in the $18 \mathrm{~S}$ gradient fractions (Input) is indicated on the left.

found in the major spliceosome.

We next asked whether any of the 12 remaining U11/U12 proteins correspond to $\mathrm{U} 1$ - or U2-specific proteins. Interestingly, the $160-, 150-, 130-$, and $49-\mathrm{kD}$ U11/U12 proteins comigrated with four of the $17 S$ U2specific proteins that constitute the essential splicing factor SF3b (that is, U2-160kD, U2$150 \mathrm{kD}, \mathrm{U} 2-120 \mathrm{kD}$, and U2-53kD) (Fig. 2) (7, 13). Moreover, antibodies directed against the U2-160kD, U2-150kD, or U2-120kD proteins reacted strongly with the 160-, 150-, and

Fig. 3. Schematic of U11/U12-35kD and U1$70 \mathrm{~K}$ proteins. The U11/ $\mathrm{U} 12-35 \mathrm{kD}$ protein is ho-

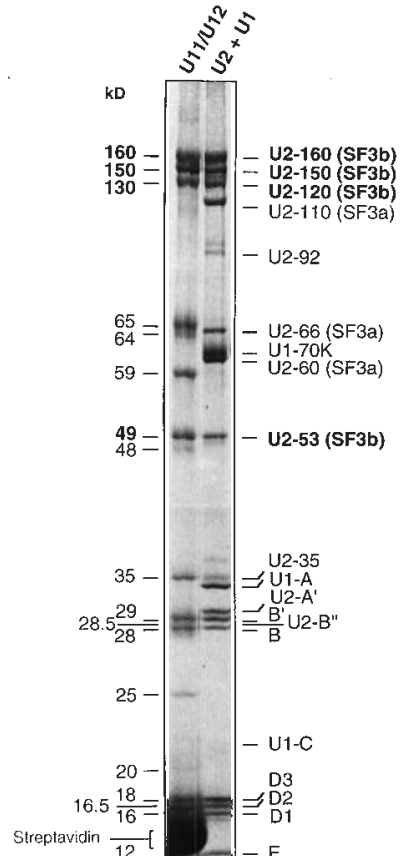

Fig. 2. SF3b proteins are present in U11/U12 snRNPs. Proteins associated with $18 S$ U11/U12 or $17 S$ U2 and $12 S$ U1 snRNPs were fractionated by SDS-PAGE and visualized by Coomassie staining.

130-kD U11/U12 proteins, respectively (12). Finally, peptide sequences of the 160-, 150-, $130-$, and 49-kD U11/U12 proteins were obtained by microsequencing (Table 1) (14). These sequences were identical to the known sequence of the corresponding SF3b protein, except for two peptides that had one or more mismatches (15). Thus, proteins that are most likely identical to the SF3b components in $17 S$ U2 snRNP are present in the U11/U12 snRNP complex. In contrast, none of the $\mathrm{U} 11 / \mathrm{U} 12$ proteins comigrated with the U2 snRNP proteins that constitute the essential splicing factor SF3a (that is, U2-110kD, U2$66 \mathrm{kD}$, and $\mathrm{U} 2-60 \mathrm{kD})(7)$. Thus, either SF3a is not a component of the U11/U12 complex, or this factor has dissociated during the U11/ $\mathrm{U} 12$ selection procedure. The U2-160kD, $\mathrm{U} 2-150 \mathrm{kD}$, and $\mathrm{U} 2-53 \mathrm{kD}$ SF3b subunits interact with U2-type introns in the vicinity of the branch site, suggesting that they are core components of the major spliceosome (1618). Similar interactions involving these same proteins may help to stabilize the association of U12 with the branch site of U12- type introns.

Protein immunoblot analysis and sequence information from six of the eight other U11/U12-specific proteins failed to identify any additional U1 or U2 snRNP proteins or intron-bridging proteins such as SF1/ mBBP or FBP21 $(19,20)$. Instead, several previously uncharacterized polypeptides were detected. However, a database search with peptide sequences obtained from one of these proteins (U11/U12-35kD) suggested that it may be related to the U1 snRNP-specific $70 \mathrm{~K}$ protein. To further characterize the U11/U12-35kD protein, we isolated a full-length cDNA (21). This cDNA encodes a 246-amino acid protein with a predicted molecular mass of $29 \mathrm{kD}$ and an isoelectric point of 9.88. A potential ortholog in Arabidopsis thaliana (GenBank accession number AC002335, PID g228900) is 47\% identical $(60 \%$ similar); this high degree of conservation between organisms as diverse as humans and plants suggests that the $35-\mathrm{kD}$ protein performs an important function. Consistent with the fact that U12-type introns are not present in Saccharomyces cerevisiae and Caenorhabditis elegans (22), 35-kD orthologs were not detected in these organisms.

The U11/U12-35kD protein contains an RNA recognition motif (amino acids 51 to 129), and this region and the adjacent glycine-rich region are the most similar between $\mathrm{U} 1-70 \mathrm{~K}$ and U11/U12-35kD (U1-70K positions 99 to 203 ; $49 \%$ identity, $65 \%$ similarity) (Fig. 3) (23). In addition, the COOH-terminal half of both proteins is rich in Arg-Asp and Arg-Glu dipeptide repeats (a so-called alternating arginine domain) (24). The similarity between these proteins suggested that they may be functional analogs. Thus, we next asked whether U11/U12-35kD is associated with U11 snRNPs. Indeed, antiserum against the $35-\mathrm{kD}$ protein specifically and efficiently immunoprecipitates U11 from a mixture of gradient-fractionated snRNPs containing U11 monoparticles (Fig. 4, lanes 3 and 4) (25). Thus, analogous to $\mathrm{U} 1-70 \mathrm{~K}$, the $\mathrm{U} 11-35 \mathrm{kD}$ protein may facilitate $5^{\prime}$ splice site recognition in the minor spliceosome. Likewise, U11-35kD may be involved in exon bridging, interacting with components of the major spliceosome bound to the pyrimidine tract of an upstream U2-type intron. Consistent with this idea, binding of the U1 snRNP to a U2-type 5' splice site enhances splicing of an upstream U12-type intron, indicating that components of both types of spliceosome can interact with each other (26).

The identification of proteins unique to mologous to the U1-70K protein. RRM, RNA recognition motif; Gly, glycine-rich; Alternating Arg (Alt Arg), rich in Arg-Asp and Arg-Glu dipeptides; Gly/Pro, glycine/proline-rich. 


\section{R E P O R T S}

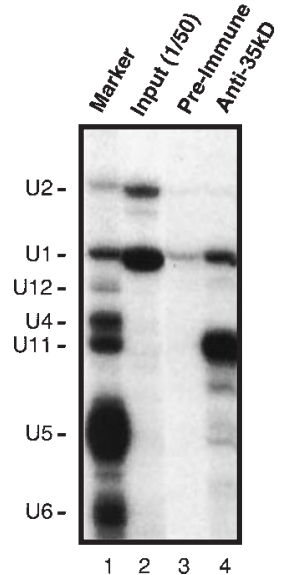

Fig. 4. The $35-\mathrm{kD}$ protein is associated with U11 snRNPs. Immunoprecipitated snRNAs were labeled with $\left[{ }^{32} \mathrm{P}\right]$ cytidine $3^{\prime}, 5^{\prime}$-bisphosphate $(P C P)$ fractionated by denaturing PAGE, and visualized by autoradiography.

the minor spliceosome is consistent with apparent differences in its mechanism of early spliceosome formation. In the major spliceosome, U1 and U2 bind to the pre-mRNA sequentially, whereas in the minor spliceosome U11 and U12 appear to bind as a preformed complex, implicating U11/U12 proteins in bridging the $5^{\prime}$ and $3^{\prime}$ ends of U12type introns (9). The identification of proteins shared by both spliceosomes (the Sm proteins and SF3b) supports proposed models for evolutionary relationships between the major and minor spliceosome $(1,22)$. Analyses of the distribution of U12-type introns in various species favor a so-called fission-fusion model in which each spliceosome (derived from a common ancestor) evolved in separate lineages before being fused in a eukaryotic progenitor (22). In this model, only one version of those proteins that did not diverge considerably before the fusion event would ultimately be retained by both spliceosomes, resulting in shared spliceosomal components. However, preliminary immunoprecipitation experiments suggest that most of the [U4/ U6.U5] tri-snRNP proteins may also be shared by both spliceosomes (27). Conservation of such a large number of proteins is more readily explained by an alternative model in which the U12-dependent spliceosome evolved in the presence of the U2dependent one. In this case, a new class of introns and snRNAs, derived from the fragmentation of a parasitic group II intron, were introduced into an organism with a preexisting spliceosome $(1,22)$. As a result of convergent evolution, facilitated by the sharing of most of the spliceosomal proteins, a distinct but similar spliceosome would be generated $(1,22)$.

\section{References and Notes}

1. C. B. Burge, T. H. Tuschl, P. A. Sharp, in The RNA World II, R. F. Gesteland and J. F. Atkins, Eds. (Cold Spring Harbor Press, Cold Spring Harbor, NY, 1999), pp. $525-560$

2. S. L. Hall and R. A. Padgett, J. Mol. Biol. 239, 357 (1994); P. A. Sharp and C. B. Burge, Cell 91, 875 (1997).

3. S. L. Hall and R. A. Padgett, Science 271, 1716 (1996); W.-Y. Tarn and J. A. Steitz, Cell 84, 801 (1996); Y.-T. Yu and J. A. Steitz, Proc. Natl. Acad. Sci. U.S.A. 94, 6030 (1997); I. Kolossova and R. A. Padgett, RNA 3 , 227 (1997).

4. W.-Y. Tarn and J. A. Steitz, Science 273, 1824 (1996)

5. R. Reed, Curr. Opin. Genet. Dev. 6, 215 (1996).

6. C. L. Will and R. Lührmann, Curr. Opin. Cell Biol. 9, 320 (1997).

7. A. Krämer, Annu. Rev. Biochem. 65, 367 (1996).

8. K. M. Wassarman and J. A. Steitz, Mol. Cell. Biol. 8, 1276 (1992)

9. M. Frilander and J. A. Steitz, Genes Dev. 13, 851 (1999).

10. Trimethylguanosine (m3G)-capped snRNPs were immunoaffinity-purified from HeLa nuclear extracts with antibodies against $\mathrm{m} 3 \mathrm{G}$ and separated on 10 to $30 \%$ glycerol gradients [B. Laggerbauer, ]. Lauber, R. Lührmann, Nucleic Acids Res. 24, 868 (1996)]. Fractions containing 185 U11/U12 snRNP complexes were pooled and the $\mathrm{KCl}$ concentration was adjusted to $250 \mathrm{mM}$. snRNPs from $2.4 \mathrm{ml}$ of pooled 185 gradient fractions were incubated for 16 hours at $4^{\circ} \mathrm{C}$ with $12 \mu \mathrm{g}$ of an oligonucleotide complementary to nucleotides 2 to 18 of human U11 snRNA, $5^{\prime}$-ACGACAGAAGCCCUUUUdT*dT*dT*dT*-3' (U11 oligo), or complementary to nucleotides 11 to 28 of human U12 SnRNA, 5'-AUUUUCCUUACUCAUAAGdT*dT*dT*dT*$3^{\prime}$ (U12 oligo), where * denotes a biotinylated $2^{\prime}$ deoxythymidine and $\mathrm{A}, \mathrm{U}, \mathrm{G}$, and $\mathrm{C}$ represent $\mathrm{2}^{\prime}-\mathrm{O}$ methyl ribonucleotides. Oligonucleotide-bound snRNPs were precipitated with streptavidin-agarose [A. I. Lamond and B. S. Sproat, in RNA Processing: A Practical Approach, D. Rickwood and B. D. Hames, Eds. (Oxford Univ. Press, Oxford, 1996), pp. 103-140]. RNA was eluted from one-fifth of the agarose-precipitated snRNPs by incubating for $30 \mathrm{~min}$ at $95^{\circ} \mathrm{C}$ in $100 \mu \mathrm{l}$ of DH buffer ( $15 \mathrm{mM} \mathrm{NaCl}, 1.5 \mathrm{mM} \mathrm{Na}$ citrate, and $0.1 \%$ SDS), fractionated on $10 \%$ polyacrylamide $-7 \mathrm{M}$ urea gels, and visualized by silver staining. The identity of the selected RNAs as U11 and U12 was confirmed by Northern blotting. Protein was eluted from the remaining beads by incubating for $5 \mathrm{~min}$ at $95^{\circ} \mathrm{C}$ in $200 \mu \mathrm{l}$ of $\mathrm{S}$ buffer $[60 \mathrm{mM}$ tris (pH 6.8), $1 \mathrm{mM}$ EDTA, $17.5 \%$ glycerol, $2 \%$ SDS, and $0.2 \mathrm{M}$ dithioerythritol (DTE)] and precipitated with five volumes of acetone. Proteins were fractionated by SDS-polyacrylamide gel electrophoresis (PAGE) on gels containing 10\% (upper half) and $13 \%$ (lower half) polyacrylamide, and visualized by Coomassie staining. For comparison, RNA and protein from $50 \mu \mathrm{l}$ of the input material (pooled 185 gradient fractions) were also analyzed.

11. C. L. Will and R. Lührmann, in Eukaryotic mRNA Processing, A. R. Krainer, Ed. (IRL Press, Oxford, 1997), p. 130.

12. C. L. Will, C. Schneider, R. Reed, R. Lührmann, unpublished data.

13. The difference in the apparent molecular mass of some of the comigrating proteins (such as U2-53kD and $\mathrm{U} 11 / \mathrm{U} 12-49 \mathrm{kD}$ ) is due to differences in the electrophoresis conditions originally used to identify the 175 U2 snRNP proteins [S.-E. Behrens et al., Mol. Cell. Biol. 13, 307 (1993)]. Note that U2-160kD, U2-150kD, U2-120kD, and U2-53kD also correspond to the spliceosomal associated proteins (SAPs) of $155,145,130$, and $49 \mathrm{kD}$, respectively.

14. Sequencing of tryptic peptides generated from each protein was performed by Toplab (Martinsried, Germany).

15. A database search with the U11/U12-130kD peptide containing three mismatches did not detect, among a total of 36 positives, any expressed sequence tags (ESTs) whose open reading frame (ORF) contained one or more of the observed amino acid changes. This indicates that $U 11 / U 12-130 \mathrm{kD}$ is probably not a splice variant of the U2-120kD protein; rather, these discrepancies most likely represent peptide sequencing errors.

16. C. Wang et al., Genes Dev. 12, 1409 (1998).

17. O. Gozani, R. Feld, R. Reed, ibid. 10, 233 (1996).

18. P. Champion-Arnaud and R. Reed, ibid. 8, 1974 (1994).

19. N. Abovich and M. Rosbash, Cell 89, 403 (1997); J. A. Berglund, K. Chua, N. Abovich, R. Reed, M. Rosbash, ibid., p. 781; M. T. Bedford, R. Reed, P. Leder, Proc. Natl. Acad. Sci. U.S.A. 95, 10602 (1998).

20. Peptide sequences have been obtained for all but the 28.5- and 20-kD U11/U12 proteins. Antibodies specific for the U1-70K, U1-A, U1-C, or U2-B" proteins did not recognize any of the U11/U12-associated proteins. Because the sequences of the U2-35kD and U2-92kD proteins are currently unknown, we cannot rigorously exclude that similar proteins are present in the U11/U12 complex. However, antibodies directed against the U11/U12-35kD protein did not recognize U2-35kD on immunoblots.

21. Peptide sequences were obtained from gel-fractionated, trypsin-digested U11/U12 proteins by microsequencing. The $35-k D$ protein peptides KEYDPLK and KRWQEREPTRVWPDND (28) were used to search the National Center for Biotechnology Information EST database for full-length cDNAs by means of the program TBLASTN. Both peptides were found in the ORF of a cDNA from human macrophage cells (GenBank accession number U44798) encoding a protein of unknown function that was noted to share homology with the U1-70K protein. An EST cDNA from human muscle cells that contains an identical ORF (GenBank accession number AA211268) was cloned and sequenced in its entirety. Protein generated by in vitro translation of this CDNA comigrated with the purified U11/U12-35kD protein on SDS-polyacrylamide gels, confirming that it encodes full-length protein (12).

22. C. B. Burge, R. A. Padgett, P. A. Sharp, Mol. Cell 2, 773 (1998).

23. See Science Online (www.sciencemag.org/feature/ data/990628.shl) for sequence alignment of the U11/ U12-35kD and U1-70K proteins.

24. K. M. Neugebauer, J. A. Stolk, M. B. Roth, J. Cell Biol. 129, 899 (1995)

25. The U11/U12-35kD protein peptide EKRWQEREPTRVWPD (positions 208 to 222) (28) was used to generate rabbit antibodies that reacted specifically with the $35-\mathrm{kD}$ protein on immunoblots (12). Immunoprecipitations were performed as described [S. Teigelkamp, C. Mundt, T. Achsel, C. L. Will, R. Lührmann, RNA 3, 1313 (1997)], except gradient-fractionated $12 S$ snRNPs were used as the input material.

26. Q. Wu and A. R. Krainer, Science 274, 1005 (1996).

27. C. Schneider and R. Lührmann, unpublished data.

28. Abbreviations for amino acid residues are as follows: A, Ala; C, Cys; D, Asp; E, Glu; F, Phe; G, Gly; H, His; I, Ile; K, Lys; L, Leu; M, Met; N, Asn; P, Pro; Q, Gln; R, Arg; $\mathrm{S}$, Ser; T, Thr; V, Val; W, Trp; and Y, Tyr.

29. B. Das, L. Xia, O. Gozani, L. Palandjian, Y. Chyung, R. Reed, unpublished data.

30. We thank G. Heyne, A. Badouin, W. Lorenz, D. Meyer, and I. Öchsner for excellent technical assistance, $M$. Krause for the synthesis of 2'-O-methyl oligonucleotides, and the Resource Center of the German Genome Project at the Max Planck Institute for Molecular Genetics, as well as the IMAGE cDNA Clone Consortium, for providing EST clones. Supported by the Gottfried Wilhelm Leibniz Program and grants from the Deutsche Forschungsgemeinschaft (A6/ SFB397) and Fonds der Chemischen Industrie (R.L.).

3 February 1999; accepted 30 April 1999 